\title{
Cáncer de próstata hormonorresistente. Cambios en las estrategias terapéuticas desde la demostración de la utilidad de la quimioterapia
}

\author{
J. Huguet Pérez, P. Maroto Rey*, J. Palou Redorta, H. Villavicencio Mavrich \\ Servicio de Urología. Fundació Puigvert. Barcelona. \\ *Servicio de Oncología del Hospital de la Santa Cruz y San Pablo. Barcelona.
}

Actas Urol Esp 2006; 30 (2): 123-133

\section{RESUMEN}

\section{CÁNCER DE PROSTATA HORMONORRESISTENTE. CAMBIOS EN LAS ESTRATEGIAS TERAPEUTICAS DESDE LA DEMOSTRACIÓN DE LA UTILIDAD DE LA GUIMIOTERAPIA}

En la década de los 90 fue dificil obtener conclusiones de las publicaciones sobre CPHR, existía una falta de homogeneidad en cuestiones tan importantes como la propia definición de CPHR, la selección de pacientes o la valoración de las respuestas a los tratamientos. En la actualidad ya existen criterios consensuados al respecto que se exponen en el trabajo.

A finales de 2004 se publicaron 2 trabajos que demostraron que la quimioterapia basada en docetaxel mejoraba la supervivencia en el CPHR con metástasis. Hasta entonces los distintos tratamientos utilizados únicamente conseguían paliación de síntomas. Pero probablemente no todos los pacientes con CPHR sean candidatos a quimioterapia con docetaxel de entrada. El debate actual se centra en determinar a qué pacientes y en qué instante debe iniciarse la quimioterapia para excluir a los que corran el riesgo de sufrir sus efectos adversos sin ventajas clínicas. Pacientes con CPHR sin metástasis pueden ser candidatos a recibir maniobras hormonales secundarias antes de iniciar quimioterapia

En esta revisión analizamos qué cambios se han producido en las estrategias terapéuticas en CPHR, desde la demostración de la utilidad de la quimioterapia, mostrando también cual es en la práctica diaria nuestra actitud en el tratamiento de estos pacientes.

Palabras clave: Cáncer de próstata hormonorresistente. Tratamiento.

\section{ABSTRACT \\ HORMONE-REFRACTORY PROSTATE CANCER. MODIFICATIONS OF THE THERAPEUTIC STRATEGIES SINCE CHEMOTHERAPY PROVED ITS USEFULNESS.}

Back in the 90's it was difficult to have access to the conclusions of publications on HRPC. Homogeneity was very scarce regarding issues as significant as the definition of HRPC itself, patient selection, or evaluation of the responses to therapy. Consensus has currently been reached on such matters, and it is described in this text.

Two works were published in late 2004 showing that docetaxel-based chemotherapy improved metastatic HRPC survival. Until then, the different treatments used could only provide symptomatic relief. But probably not all of the HRPC patients are eligible for primary docetaxel chemotherapy. The current debate focuses on determinating to which patients should chemotherapy be administered and at which time should it start, in order to exclude those patients at risk of experiencing its adverse effects without benefitting from its clinical advantages. Non-metastatic HRPC patients may be candidates to receiving secondary hormone manoeuvres before starting with chemotherapy.

We will analyse in this review the changes occurred in the therapeutic strategies ever since chemotherapy showed its value, and we shall also disclose our attitude regarding treatment of these patients in daily practice. 


\section{LA HETEROGENEIDAD DEL CÁNCER DE PRÓSTATA HORMONORRESISTENTE}

Higgins y Hodges observaron que la castración y el tratamiento con dietilestilbestrol eran efectivos en el tratamiento del cáncer de próstata metastásico ${ }^{1}$. La reducción de los niveles de testosterona con orquiectomía o castración médica fueron desde entonces la terapia aplicada en pacientes con enfermedad diseminada.

La deprivación androgénica (DA) es un tratamiento paliativo que inicialmente consigue respuestas objetivas y subjetivas entre el 60\% y $80 \%$ de los casos. La duración de la respuesta es variable $\mathrm{y}$ ha permanecido estable durante las cinco últimas décadas. El tiempo hasta la progresión clínica de los pacientes con cáncer de próstata metastásico varía entre 18 y 24 meses y la supervivencia entre 24 y 36 meses $^{2,3}$. A pesar de la buena respuesta inicial, prácticamente todos los pacientes evolucionan a un estado de hormonorresistencia. En esta situación la supervivencia oscila entre 7 y 16 meses $^{4,5}$.

El cáncer de próstata hormonorresistente (CPHR) presenta una importante heterogeneidad. El comportamiento clínico, la respuesta a tratamientos y la supervivencia varía dependiendo de subgrupos de pacientes. Casos que muestran únicamente una elevación del antígeno prostático específico (PSA) tras la DA difieren notablemente de otros con elevación del PSA y enfermedad metastásica (partes blandas o hueso), de escaso o importante volumen, y con o sin sintomas. Esta variabilidad clínica, unida a los históricamente poco consensuados criterios de selección de pacientes y de valoración de respuestas que se apreciaba en la literatura, hicieron difícil en ocasiones evaluar la utilidad de los tratamientos $^{5-7}$. A finales del 2004 se publicaron 2 trabajos que demostraron que la quimioterapia basada en docetaxel mejoraba la supervivencia en pacientes con $\mathrm{CPHR}^{8,9}$. Hasta entonces los distintos tratamientos utilizados únicamente conseguian paliación de síntomas.

En el presente trabajo hacemos una revisión sobre el CPHR, para analizar posteriormente qué cambios se han producido en las estrategias terapéuticas desde la demostración de la utilidad de la quimioterapia. En la parte final mostramos cual es en la práctica diaria nuestra la actitud en el tratamiento de estos pacientes. En este trabajo no se analizan los numerosos fármacos en fase de investigación que existen en la actualidad para el tratamiento del CPHR.

\section{DEFINICIÓN DE CPHR}

Históricamente se consideraba que un paciente tenía un CPHR cuando presentaba una progresión clínica o por PSA en presencia de una correcta DA. Al observarse que era posible una respuesta a maniobras hormonales secundarias (retirada del antiandrógeno, antiandrógeno secundario, estrógenos, ketoconazol, etc.) pasó a considerarse tumor hormonorresistente aquel que ya no respondía a tales maniobras ${ }^{10}$.

Con el tiempo se han ido estableciendo una serie de criterios consensuados de inclusión de pacientes en ensayos clínicos lo que ha ayudado a homogeneizar el concepto de CPHR.

En la actualidad se considera que existe progresión tras la DA cuando aparecen nuevas lesiones óseas, adenopáticas o en partes blandas, o también, ante un aumento del PSA con concentraciones de testosterona en niveles de castración ( $<50 \mathrm{ng} / \mathrm{ml}$ ). Todo ello después de la retirada del antiandrógeno como mínimo 4-6 semanas. La progresión bioquímica se define como 2 aumentos del PSA superiores al 50\% respecto al nadir (determinaciones separadas como mínimo 2 semanas). También se aconseja que el PSA sea superior a $5 \mu \mathrm{g} / \mathrm{L}$ si se plantea incluir al paciente en un estudio ${ }^{11}$.

\section{FACTORES PRONÓSTICOS EN EL CPHR}

La heterogeneidad del CPHR hace preciso valorar factores pronósticos para crear grupos de riesgo. Esto permitiría seleccionar los tratamientos más adecuados para cada paciente, sobre todo en la actualidad en que se amplian las opciones terapéuticas. Son escasos los factores pronósticos valorados en trabajos prospectivos y bien diseñados.

Nivel de actividad: Las escalas más utilizadas para la valoración del nivel de actividad han sido las del Eastern Cooperative Oncology Group (ECOG) con unos valores entre 0 y 5 (el 0 es la categoría con mejor nivel de actividad) y la de 
Karnofsky con valores entre 0 y 100\% (el 100\% es el mejor nivel de salud). Tiene el inconveniente de la subjetividad. Se ha observado un peor pronóstico de forma significativa en pacientes con CPHR y ECOG $<2$ o Karnofsky $<80 \% \%^{5,12}$.

Hemoglobina: A pesar de que los pacientes con DA presentan una reducción del 15\% de la hemoglobina (Hb), cifras inferiores a 10-12 g/dl se asocian a un peor pronóstico de forma independiente. Niveles de $\mathrm{Hb}$ inferiores a $12 \mathrm{~g} / \mathrm{dl}$ asociados a fatiga y PSA superior a $100 \mu \mathrm{g} / \mathrm{L}$ se han correlacionado con una peor supervivencia. Se ha objetivado la importancia de los niveles bajos de $\mathrm{Hb}$ como factor pronóstico cuando se asocian a un escaso nivel de actividad. El impacto negativo de la disminución de $\mathrm{Hb}$ se ha relacionado con la afinidad de las metástasis óseas por la médula ósea roja, estando el grado de anemia acorde con la extensión de la enfermedad ósea ${ }^{5,12}$.

Lactato deshidrogenasa: El enzima lactato deshidrogenasa (LDH) se libera a la sangre a partir de diferentes tejidos, principalmente el hígado. Se considera un marcador de enfermedad metastásica visceral y su elevación no sólo aparece en pacientes con metástasis hepáticas. Valores elevados implican un peor pronóstico.

Aunque estudios han sugerido que la afectación metastásica visceral se asocia a un peor pronóstico, otros no observan diferencias en la supervivencia respecto a la afectación ósea ${ }^{5,12}$.

PSA: En los pacientes con CPHR existe discusión sobre el valor del PSA como factor pronóstico. En estos pacientes parece que la expresión del PSA es inferior a la de los tumores hormonosensibles y puede no correlacionarse con la proliferación celular. No se ha logrado identificar los valores del PSA a partir de los cuales el pronóstico es peor. Se ha observado que pacientes con CPHR y PSA superior a 100-150 $\mu \mathrm{g} / \mathrm{L}$ antes de iniciar un nuevo tratamiento, tienen una supervivencia menor, y todavía disminuye más, si se asocia a unos niveles de hemoglobina inferior a $12 \mathrm{~g} / \mathrm{dl}^{5,13}$. Otros han observado que un periodo de duplicación del PSA superior a 80 días se asocia a mejores resultados terapéuticos ${ }^{14}$.
La pérdida de peso también se ha estudiado pero su valor está por definir. Los estudios de la extensión de la enfermedad valorada con la cuantificación de las metástasis mediante radiografias, gammagrafias y niveles de fosfatasa alcalina son de notable interés pero es difícil obtener conclusiones por la gran disparidad de variables estudiadas. Se han descrito también factores de riesgo moleculares como la presencia de marcadores de diferenciación neuroendocrina, hecho este que le confiere al tumor un crecimiento andrógeno-independiente. La demostración de la amplificación del receptor androgénico en biopsias de próstata de pacientes tras progresión, se ha asociado a una mejor respuesta a una terapia de segunda línea ${ }^{12}$.

\section{VALORACIÓN DE LA RESPUESTA AL TRATAMIENTO EN EL CPHR}

En el CPHR no son útiles los clásicos criterios de respuesta completa, respuesta parcial, enfermedad estable y enfermedad en progresión. Esto se debe a que entre el $80-90 \%$ de los pacientes no tienen enfermedad medible bidimensionalmente mediante pruebas de imagen. Además las metástasis óseas, las más frecuentes, son de difícil cuantificación. El efecto llamarada en el que puede haber un incremento de la intensidad de las lesiones óseas al iniciar el tratamiento, las respuestas mixtas, en las que la regresión de algunas metástasis óseas se acompañan de la progresión de otras, y la variabilidad interindividual en interpretación de las exploraciones, hace que no sean utilizadas sistemáticamente para la valoración de la respuesta a los tratamientos. Estas particularidades del CPHR provocan que la valoración de la respuesta al tratamiento deba hacerse objetivando cambios en el PSA, en la enfermedad medible cuando es posible y en los sintomas ${ }^{5,15}$.

Cambios en el PSA: Se ha observado una mejor supervivencia en aquellos pacientes con CPHR y un descenso de los niveles de PSA superiores al $50 \%$ después del tratamiento. En la actualidad este descenso del 50\% del PSA se considera criterio de respuesta tras una determinación de confirmación a las 4 semanas de la primera ${ }^{16}$. A pesar de ello también existe discusión sobre el 
papel del PSA como marcador de respuesta. En trabajos sobre pacientes con CPHR y enfermedad medible, la respuesta definida como un descenso superior al 50\% del PSA, se correlacionaba con un $68 \%$ de respuesta en la enfermedad medible. Por lo tanto el descenso del PSA no siempre se acompaña de un descenso de la actividad tumoral. Sí se acepta que la disminución de los niveles del PSA comporta algún efecto biológico y por esto se considera de utilidad para hacer una validación de la actividad de nuevos agentes ${ }^{5,12,17}$.

Cambios en la enfermedad medible: Se consideran lesiones medibles aquellas que pueden cuantificarse por técnicas radiológicas convencionales. Las lesiones diana son lesiones medibles representativas de los órganos afectos. Una respuesta completa al tratamiento implica una desaparición de las lesiones diana. En una respuesta parcial se observa una disminución de al menos un $30 \%$ de la suma de los diámetros mayores iniciales de la lesión diana y la progresión supondría el aumento del $20 \%$ de los diámetros de las lesiones o la aparición de nuevas ${ }^{5}$.

Calidad de vida: En el tratamiento del CPHR, la mejoría de la calidad de vida con el control del dolor y los síntomas adquiere considerable importancia. Los instrumentos utilizados para valorar mejoras en la calidad de vida son los cuestionarios.

Una vez aplicado el tratamiento en pacientes con CPHR, se consideran habitualmente criterios de progresión, el aumento de tamaño o aparición de nuevas lesiones medibles, nuevas áreas de captación en radiografias simples o gammagrafias óseas o un aumento de los valores del PSA.

En trabajos recientes se ha definido enfermedad progresiva, además de a la aparición de nuevas lesiones medibles, al incremento del $25 \%$ del valor del PSA (hasta al menos $5 \mathrm{ng} / \mathrm{ml}$ ) respecto al PSA pre-tratamiento y en casos que presentan durante el tratamiento una disminución del PSA, al aumento del 25\% del mismo (hasta al menos $5 \mathrm{ng} / \mathrm{ml}$ ) por encima del nadir logrado ${ }^{8}$.

\section{TRATAMIENTO DEL CPHR}

Ante el ascenso del PSA en un paciente con DA, debe comprobarse que los niveles de testos- terona sean de castración. Se ha descrito hasta un $11 \%$ de niveles de testosterona superiores a los deseados a pesar de la orquiectomía ${ }^{18}$.

La siguiente cuestión que se plantea en el tratamiento del CPHR es si debe mantenerse la DA. La mayoría de los autores aconsejan seguir la DA a pesar de que los beneficios parecen ser de escasa cuantía. Se fundamentan en que se ha demostrado que la administración de andrógenos a pacientes con $\mathrm{CP}$ metastásico estimula el crecimiento tumoral. En el CPHR la suspensión de la DA podría inducir el crecimiento del tumor por la posibilidad de persistir células andrógenosensibles ${ }^{5,17,19,20}$. Son precisos estudios prospectivos respondiendo esta cuestión porque también están implicados importantes factores económicos.

\section{MANIOBRAS HORMONALES SECUNDARIAS}

Retirada del antiandrógeno

La mayoria de los pacientes con CPHR ha recibido antiandrógenos. De entrada, constituyendo el BAC (castración médica o quirúrgica más antiandrógeno), o posterior a monoterapia (castración médica o quirúrgica sin antiandrógeno). En la actualidad se considera que el primer paso a realizar ante una la elevación del PSA en un paciente con DA que incluya antiandrógenos es la retirada de los mismos ${ }^{19-21}$. Esta maniobra provoca descensos del PSA superiores al 50\% en el 11-40\% de los casos, manteniéndose la respuesta entre 4 y 6 meses. Estos descensos del PSA se han asociado en ocasiones a mejoría subjetiva y sintomática ${ }^{22-24}$. La retirada del antiandrógeno (RA) se describió inicialmente para la flutamida pero se ha observado también con bicalutamida $^{25}$, nilutamida ${ }^{26}$, acetato de megestrol ${ }^{27} \mathrm{e}$ incluso con dietilestilbestrol ${ }^{28}$. El descenso de los valores del PSA se objetiva en las 4 semanas siguientes a la retirada de la flutamida, prolongándose hasta 7 en el caso de la bicalutamida por tener una mayor vida media. La duración del tratamiento previo con antiandrógeno parece ser el indicador más útil para predecir la respuesta a esta maniobra ${ }^{19}$, aunque se han descrito respuestas en pacientes tratados con antiandrógenos únicamente 2 meses $^{29}$. Es difícil saber el impacto real de esta maniobra en el tratamiento 
del CPHR debido a que en los distintos trabajos existen numerosas intervenciones terapéuticas previas y posteriores a la RA causantes de confusión. No se ha demostrado que la RA prolongue la supervivencia y no parece predecir la respuesta a otras maniobras hormonales posteriores.

Debido a las aceptables tasas de respuesta, se aconseja que los pacientes con progresión tras DA deben intentar la RA. Es preciso también realizar esta maniobra al incluir pacientes en estudios para evitar confusiones al valorar las respuestas a los fármacos. Se cree que el mecanismo responsable de este fenómeno es el de la aparición de mutaciones en el receptor androgénico, de forma que los antiandrógenos podrian estimular el receptor en lugar de inhibirlo ${ }^{5,17,19-21}$.

\section{Antiandrógeno diferido}

Los pacientes con cáncer de próstata avanzado que progresan tras haber recibido tratamiento con monoterapia pueden beneficiarse de la administración de un antiandrógeno. Sobre el 50\% de los casos se producen descensos significativos de los niveles del PSA, reduciéndose al 23\% en pacientes sintomáticos. La respuesta a la retirada del antiandrógeno cuando el antiandrógeno es utilizado como maniobra hormonal secundaria es inferior en comparación a cuando se utiliza de inicio $^{19,30}$.

\section{Antiandrógenos secundarios}

A pesar de sus similitudes, se cree que los antiandrógenos pueden actuar de distinta forma a nivel del receptor androgénico. Esto podría ir a favor de que no hubiese resistencias cruzadas entre ellos y que la administración de un antiandrógeno diferente fuese efectivo. Se ha observado que el tratamiento con bicalutamida a dosis altas (150-200 mg / $24 \mathrm{~h}$ ) produce una respuesta en los niveles del PSA entre el 22-38\% de los casos, sobre todo, en pacientes que han recibido previamente flutamida. No se han observado respuestas medibles pero sí mejoras en el control del dolor. La respuesta es prácticamente nula al administrar bicalutamida tras dos o más maniobras hormonales. Es un tratamiento bien tolerado siendo el efecto secundario principal los sofo$\cos \mathrm{y}$ ginecomastia ${ }^{31,32}$.
El acetato de megestrol se ha utilizado con escaso éxito en esta situación ${ }^{33}$ y está por demostrar la efectividad de la flutamida tras la retirada de la bicalutamida y papel del acetato de ciproterona y la nilutamida como antiandrógenos secundarios $^{19}$.

\section{Inhibidores de los andrógenos suprarrenales}

Se considera que cerca del 10\% de los andrógenos circulantes se segregan en las glándulas suprarrenales (SSRR). En estados andrógenoindependientes algunas células tumorales podrían mantener sensibilidad a pequeñas cantidades de andrógenos y por tanto su reducción podría inducir respuestas. La aminoglutetimida, el ketoconazol y los corticoides son los fármacos más utilizados.

Aminoglutetimida: Actúa bloqueando diversos pasos de la hidroxilación de los esteroides induciendo una suprarrenalectomía funcional. Se administra a dosis entre $1.000 \mathrm{mg}$ y $1.750 \mathrm{mg}$ cada $24 \mathrm{~h}$ y asociada a corticoides para evitar la insuficiencia suprarrenal. Estudios valorando descensos del PSA superiores al 50\% han observado respuestas del $48 \%$ en pacientes tratados con aminoglutetimida más hidrocortisona tras la supresión antiandrogénica y del 65\% al administrar esta combinación al mismo tiempo que la retirada de la flutamida. Los efectos adversos de este fármaco limitan su uso. Aparece con frecuencia hipotensión ortostática, fatiga, ataxia y rash cutáneo ${ }^{20,34}$.

Ketoconazol: Es un imidazol con efectos antifúngicos y antiandrogénicos. Inhibe las enzimas dependientes del sistema del citocromo P-450 que participan en la esteroidogénesis gonadal y adrenal. Los efectos del ketoconazol son dosis dependientes y reversibles. Se administra vía oral a dosis de $400 \mathrm{mg} / 8 \mathrm{~h}$. Es aconsejable asociar prednisona (10 mg/24h) o hidrocortisona (20-30 $\mathrm{mg} / 24 \mathrm{~h}$ ) para evitar una insuficiencia suprarrenal. Se han observado descensos del PSA superiores al $50 \%$ entre el $27 \%$ y $60 \%$ de los pacientes tratados con ketoconazol a dosis altas $(400$ $\mathrm{mg} / 8 \mathrm{~h}$ ) y retirada del antiandrógeno, con una duración media de la respuesta entre 3,5 y 5 meses. Los efectos secundarios más comunes son las náuseas y vómitos que aparecen entre el 
$15-27 \%$ de los casos y habitualmente son tolerables. Ocasionalmente aparecen elevaciones transitorias de la transaminasas de escasa repercusión. El fármaco requiere un $\mathrm{pH}$ ácido para una correcta absorción. Por lo tanto no debe administrarse con antiácidos, bloqueantes $\mathrm{H}_{2}$ e inhibidores de la bomba de protones ${ }^{35,36}$. A mitad de dosis $(200 \mathrm{mg} / 8 \mathrm{~h})$ se han observado respuestas equivalentes $\mathrm{y}$ con menor toxicidad ${ }^{37}$. En un reciente estudio con 79 pacientes y un seguimiento prolongado, se observó un descenso del PSA superior al $75 \%$ en el $44 \%$ de los casos. Pacientes que respondían al tratamiento y con mínima o sin afectación metastásica presentaron en ocasiones respuestas prolongadas (entre 17 meses y más de 7 años $^{38}$ ).

Corticoides: Inhiben la producción hipofisaria de ACTH, provocando un descenso de la secreción esteroidea adrenal. Se han utilizado durante décadas como medida paliativa. La prednisona a dosis bajas (7,5 mg-15 mg/24h) reduce el dolor y mejora la calidad de vida entre el $40-80 \%$ de los pacientes. Los corticoides inducen un aumento de peso y mejoría del apetito y estado general. En pacientes con CPHR tratados con prednisona $(7,5$ mg-15 mg) o hidrocortisona (30-40 mg/24h) se han observado descensos significativos de los valores del PSA entre el $15-35 \%$ de los casos. Esta respuesta debe tenerse en cuenta al valorar la efectividad de fármacos que se administran conjuntamente con corticoides ${ }^{39,40}$.

Estrógenos: Actúan inhibiendo la producción de gonadotrofinas hipofisarias, la producción adrenal de andrógenos y tienen también actividad citotóxica. El dietilestilbestrol (DES) a dosis bajas $(1 \mathrm{mg} / 24 \mathrm{~h})$ provoca descensos significativos del PSA en el $43 \%$ de los pacientes con fracaso al tratamiento hormonal ${ }^{41}$. Dosis superiores no parecen justificadas por el elevado porcentaje de efectos indeseables principalmente cardiovasculares. Con el fosfestrol (difosfato de dietilestilbestrol) vía oral a dosis de $100 \mathrm{mg} / 8 \mathrm{~h}$ se han observado respuestas de descenso del PSA sobre el $75-80 \%$ de los casos con una duración media de 7 meses $^{42}$. Se han descrito mejorías subjetivas y descensos significativos del PSA en el 30\% de los casos en trabajos utilizando el fosfestrol endovenoso a dosis de $1 \mathrm{~g} / 24$ horas entre 5 y 7 días $^{43}$. Otros no corroboran estos hallazgos e incluso desaconsejan este tratamiento por su considerable morbilidad y escasa mejoría clínica ${ }^{44}$.

El inconveniente principal de los estrógenos son las complicaciones cardiovasculares (8-12\%), en ocasiones graves, que a dosis bajas disminuyen pero no desaparecen. Las complicaciones son mas frecuentes en mayores de 75 años, con sobrepeso y antecedentes de patología cardiaca o vascular $^{41}$. El estrógeno vía oral llega al hígado por la circulación portal y provoca una disminución de la síntesis de antitrombina III y un aumento del factor VII originando un estado de hipercoagulabilidad. La administración parenteral se estrógenos evitaría este fenómeno de primer paso a través del hígado. Hay un interesante estudio con estrógenos intramusculares que han demostrado una disminución de efectos adver$\operatorname{sos}^{45}$.

Una de las controversias actuales es saber qué papel tienen ahora las maniobras hormonales secundarias. Con anterioridad, el hecho de no haber ningún ensayo clínico que demostrara que la quimioterapia prolongaba la supervivencia en pacientes con CPHR, hacía que las maniobras hormonales secundarias estuviesen justificadas. Se utilizaban en pacientes con signos bioquímicos de progresión tras la RA y preferentemente con poca sintomatología. Al fracasar las maniobras hormonales secundarias se valoraba la posibilidad de administrar quimioterapia.

El papel actual de estos tratamientos se discutirá al analizar el papel de la quimioterapia en el CPHR.

\section{QUIMIOTERAPIA EN EL CÁNCER DE PRÓSTATA}

Históricamente parecía establecida la quimioresistencia del cáncer de próstata. Esto dificultó el desarrollo de la quimioterapia por considerarse no justificada su toxicidad asociada, Yagoda et al, en una revisión de 26 publicaciones sobre quimioterapia en cáncer de próstata entre 1988 y 1991, observaron una tasa de respuestas objetivas del $8,7 \%$, sin efectos en la supervivencia ${ }^{46}$.

En 1996 la FDA autorizó la asociación Mitoxantrone+prednisona para el tratamiento 
paliativo del CPHR. Mitoxantrone $\left(12 \mathrm{mg} / \mathrm{m}^{2}\right.$ cada 3 semanas) en combinación con prednisona (10 mg/dia) demostró un beneficio significativo en la paliación de síntomas respecto a prednisona sola. Se observó una reducción en las escalas de dolor y la no necesidad de aumentar el uso de analgesia en el 29\% de los pacientes con mitoxantrone respecto al $12 \%$ con prednisona ( $<<0,0001)$. La duración de la paliación fue mayor también en la rama de mitoxantrone (43 vs 18 semanas, $(\mathrm{p}<0,0001)$. El tratamiento fue bien tolerado pero no evidenció una mejoría en la supervivencia. Sólo basado en estos parcos resultados, hasta la actualidad, mitoxantrone y prednisona se consideraba el tratamiento estándar con quimioterapia de los pacientes con $\mathrm{CPHR}^{47}$.

En nuestro medio se utilizaba con frecuencia el fosfato de estramustina en monoterapia, uno de los primeros fármacos aprobados por la FDA (1981) para el tratamiento del CPHR. Es un compuesto de estradiol y una mostaza nitrogenada que actúa por unión a proteinas microtubulares y teóricamente, por su diseño, con selectividad sobre la célula tumoral. Al desagregar los microtúbulos inhibe la mitosis, pero a diferencia de los citostáticos clásicos, no provoca toxicidad hematológica y sus efectos antitumorales no parecen relacionarse directamente con su componente citostático. A pesar de que algún estudio había objetivado tasas de respuesta del $37-67 \% 48,49$, su actividad en monoterapia se consideraba limitada.

El interés por la quimioterapia en el cáncer de próstata se incrementó progresivamente a lo largo de los años 90 a partir de diferentes hallazgos in vitro que se tradujeron en resultados en la práctica clínica. Así, se evidenció la importancia del oncogén bcl-2 en los mecanismos que median el proceso de crecimiento de la célula prostática en situación de independencia de andrógenos. Este oncogén se encuentra amplificado progresivamente a medida que el cáncer de próstata se hace hormonorrefractario y además media en los mecanismos de estabilidad del microtúbulo. Esto sugería que citostáticos que actuasen a ese nivel, como ya se mostraba de manera muy segmentaria con el fosfato de estramustina, podrían ser activos en ese contexto. Citostáticos antimicrotúbulo que se utilizaron en pequeños ensayos fase
II en combinación con fosfato de estramustina fueron la vinblastina y la vinorrelbina ${ }^{50,51}$ que ya superaban con creces las tasas de respuesta que proporcionaba mitoxantrone en monoterapia.

El siguiente avance significativo ocurrió con la aplicación de los taxanos en el tratamiento de la enfermedad hormonorrefractaria. Los taxanos son citostáticos de última generación desarrollados en la década de los 80 y utilizados en clínica en el cáncer de mama y ovario a principios de los 90. Son fármacos que actúan a nivel de los microtúbulos y que parece que pueden promover la apoptosis a través de la inducción de la fosforilación de bcl-2. El taxano más representativo, el paclitaxel, obtuvo un 39\% de respuestas por PSA pero con una toxicidad elevada ${ }^{52}$. Docetaxel, un taxano de segunda generación desarrollado prácticamente en paralelo, demostró en varios estudios descensos del PSA en el 38-48\% de los casos con una mediana de supervivencia entre 9 y 18 meses y una toxicidad aceptable ${ }^{53-55}$. La combinación de estos fármacos con fosfato de estramustina pareció potenciar su actividad en estudios fase II, aumentando aparentemente la tasa de respuestas con respecto a la monoterapia ${ }^{56}$.

El siguiente paso fue la comparación de estos citostáticos con el tratamiento más eficaz hasta la fecha que era el mitoxantrone. De los dos taxanos sería el docetaxel, el que con una mayor tasa de respuestas en ensayos fase II, se acabaría desarrollando completamente en el tratamiento de pacientes con CPHR. Dos fueron los ensayos en fase III en que se compararon mitoxantrone con docetaxel, uno americano (SWOG) y un segundo canadiense y europeo (TAX).

En el estudio SWOG $9916^{8}, 674$ pacientes con CPHR metastásico se aleatorizaron a recibir docetaxel $(60 \mathrm{mg} / \mathrm{m} 2$ cada 3 semanas) más estramustina $(280 \mathrm{mg} / 8 \mathrm{~h}$ en dias del 1 al 5) o mitoxantrone más prednisona a dosis estándar. El 90\% de los pacientes tenían un buen estado general (ECOG 0-1). Únicamente un 18\% de los casos presentaban progresión definida por elevación del PSA. En los restantes, la progresión de la enfermedad era medible, o no medible pero evaluable (p.e gammagrafía ósea). La tasa de respuestas por PSA fueron superiores en el esquema con docetaxel/estramustina $(50 \%$ vs $27 \%$, $\mathrm{p}<0,0001)$. La supervivencia libre de progresión 
fue de 6,3 meses en pacientes con docetaxel/ estramustina comparado con 3,2 meses en el esquema con mitoxantrone $(\mathrm{p}<0,001)$. La supervivencia mediana fue de 17,5 meses en el grupo tratado con docetaxel/estramustina y de 15,6 meses con mitoxantrone y prednisona $(p=0,02)$. En pacientes tratados con docetaxel/estramustina se observó un incremento de los episodios de fiebre neutropénica, toxicidad cardiovascular, náuseas alteraciones metabólicas y neurotoxicidad. Un $15 \%$ de los pacientes tratados con docetaxel y estramustina presentaron complicaciones cardiovasculares y tromboembólicas que llevaron a la recomendación de utilizar aspirina $325 \mathrm{mg}$ y warfarina $2 \mathrm{mg}$ en este grupo. Esta profilaxis no pareció reducir las complicaciones cardiovasculares.

En el estudio TAX $327^{9}, 1.006$ pacientes con CPHR metastásico fueron aleatorizados a recibir docetaxel $75 \mathrm{mg} / \mathrm{m}^{2}$ cada 3 semanas vs docetaxel semanal $\left(30 \mathrm{mg} / \mathrm{m}^{2}\right.$ en ciclos de 5 dosis cada 6 semanas) vs el esquema estándar de mitoxantrone. En los tres esquemas se administró prednisona $5 \mathrm{mg}$ dos dosis diarias. Las características de los pacientes eran similares al estudio SWOG 9916. Sobre el $85 \%$ de los pacientes tenían un Karnofsky superior al 80\% y el 90\% metástasis óseas. La tasa de respuestas por PSA fue del 45$48 \%$ en tratados con docetaxel y del 32\% en los tratados con mitoxantrone $(\mathrm{p}<0,001)$. Un hecho relevante fue la mejoría significativa en el control del dolor y en la calidad de vida observada en los pacientes tratados con los dos esquemas de docetaxel respecto a mitoxantrone (22\% vs $13 \%$ ). La supervivencia mediana de los pacientes tratados con docetaxel fue superior a la observada con mitoxantrone. Estas diferencias fueron más significativas con el esquema de docetaxel cada 3 semanas ( 18,9 vs 16,5 meses, $p=0,009)$. Las diferencias con docetaxel semanal no alcanzaron la significación estadística. (17,4 vs 16,5 meses, $\mathrm{p}=0,36)$. La toxicidad más importante fue la neutropenia grado III-IV que apareció en el 32\%, $1,5 \%$ y $22 \%$ de los pacientes tratados con docetaxel cada 3 semanas, docetaxel semanal y mitoxantrone respectivamente. La toxicidad no hematológica fue superior en los pacientes con docetaxel, aunque la incidencia de toxicidad grado III-IV fue inferior al 5\% en los 3 brazos de tratamiento.
Los efectos adversos no hematológicos en los dos esquemas con docetaxel fueron comparables y los más frecuentes incluyeron alopecia (50-65\%), fatiga (49-53\%), náusea (36-41\%), diarrea (32$34 \%)$ y neuropatía (24-30\%).

\section{Análisis de los resultados de los estudios SWOG 99 y TAX 327}

Lo más remarcable de ambos estudios es el hallazgo consistente de que el docetaxel administrado cada 3 semanas es superior al mitoxantrone. Docetaxel es el primer quimioterápico con el que se ha observado una mejoría de la supervivencia en pacientes con CPHR avanzado con una toxicidad aceptable. En la actualidad se considera el tratamiento estándar en esas situaciones. Aunque para demostrarlo científicamente serían necesarios estudios aleatorizados con un elevado número de pacientes, la adición de fosfato de estramustina parece no ofrecer ningún beneficio clínico. Esto, unido a la toxicidad cardiovascular que se asocia a su uso, ha hecho que hoy por hoy no se aconseje su asociación a docetaxel ${ }^{57}$.

Otros analizan los resultados de forma crítica, e indican que responden a docetaxel sólo un tercio de los pacientes. Únicamente un 9\% por encima de las pautas estándar con mitoxantrone, a expensas de más efectos adversos y costes, con un beneficio escaso en supervivencia (2,4 meses). Añaden que los resultados se obtienen en pacientes con medianas de edad entre 68 y 69 años y mayoritariamente con buen estado general. Pacientes que no siempre corresponden a lo que se encuentra el urólogo en el día a día, añosos y con comorbilidades asociadas ${ }^{58}$. Todo ello sólo refuerza, de hecho, la importancia de la correcta selección de los posibles candidatos a un tratamiento citostático en este contexto. Es también evidente que por primera vez un fármaco citostático ha conseguido mejorar la supervivencia en pacientes con CPHR metástasico. Como casi todas las enfermedades neoplásicas, y quizás aun más en el CPHR, estamos tratando una enfermedad muy heterogénea, con comportamientos en ocasiones indolentes y oligosintomáticos durante años y en otras, con enfermedad rápidamente progresiva y de control sintomático difícil. Sistematizar esto en grupos de pacientes es complejo. 
El debate actual se centra en determinar a qué pacientes y en qué instante debe iniciarse la quimioterapia. No se tiene evidencia de si la quimioterapia administrada en estadios iniciales del CPHR (primer aumento del PSA sin metástasis) sea superior o inferior a un tratamiento más diferido. Por lo tanto es fundamental para el urólogo y oncólogo identificar el paciente que pueda beneficiarse del tratamiento con quimioterapia y excluir a aquellos que corren el riesgo de sufrir los efectos adversos de este tratamiento sin ventajas clínicas.

En nuestro centro existe una estrecha colaboración con el Servicio de Oncología del Hospital de la Santa Cruz y San pablo, y ya en el año 2000 se contemplaba en nuestros protocolos la opción de la quimioterapia con docetaxel en pacientes con CPHR. Los resultados de los primeros 23 pacientes con CPHR y síntomas que recibieron quimioterapia basada en docetaxel entre noviembre de 2000 y noviembre 2002 se presentaron en reuniones nacionales ${ }^{59,60}$. Se observó un $43 \%$ de respuestas por PSA con una mediana de duración de 6 meses y 9 meses de control sintomático. En la actualidad hay más de 70 pacientes tratados con docetaxel en colaboración con el Servicio de Oncología antes citado. Todo esto nos ha permitido ir elaborando unas pautas generales de actuación en los pacientes con CPHR en espera de que se solucionen las numerosas incógnitas que quedan por resolver en el tratamiento de este grupo de pacientes.

A efectos prácticos, cuando un paciente presenta una elevación del PSA a pesar de una correcta deprivación androgénica, se retira el antiandrógeno si realiza bloqueo androgénico completo (BAC) o se añade si realiza monoterapia con análogos de la LHRH. Si de nuevo aumenta el PSA se evalúa la extensión tumoral mediante TAC y gammagrafía ósea. Si únicamente existe una elevación del PSA pueden valorarse maniobras hormonales secundarias con ketoconazol, bicalutamida a dosis altas, estrógenos (en pacientes sin riesgo cardiovascular) o también utilizar fosfato de estramustina en monoterapia. Si el paciente está sintomático, presenta enfermedad metastásica importante o un tiempo de doblaje de PSA rápido (inferior a 2 meses) lo remitimos a oncología directamente para iniciar quimioterapia. Una vez en el servicio de oncología los pacientes recibirán tratamiento con docetaxel $70 \mathrm{mg} / \mathrm{m}^{2}$ cada 3 semanas con prednisona $50 \mathrm{mg} 2$ días para evitar reacciones adversas. No se asocia estramustina y no se retiran los análogos de la LHRH. Reciben una mediana de 6 ciclos de quimioterapia. En general recomendamos, si la toxicidad es aceptable, completar al menos 3 ciclos de tratamiento antes de valorar como no respondedor a un paciente, ya que en nuestra serie, hasta un $20 \%$ de los casos no presentaron descensos apreciables del PSA antes del segundo ciclo. Habitualmente la mejoría clínica en los respondedores es evidente ya desde el primer tratamiento. En caso de buena respuesta se prosigue hasta completar 6-8 ciclos. El seguimiento se realiza mediante PSA y TAC y gammagrafía ósea si hay enfermedad medible. $\mathrm{Ni}$ la edad ni la insuficiencia renal son contraindicaciones para el tratamiento, si bien, en pacientes muy frágiles se considera la utilización de la pauta semanal, menos tóxica. Cuando existe progresión de la enfermedad un $25 \%$ de los pacientes, habitualmente respondedores, pueden volver a recibir la misma pauta de quimioterapia. Finalmente, cuando existe evidencia de resistencia a la quimioterapia, los pacientes reciben tratamiento de soporte o entran en un protocolo de estudio clínico. Rara vez se ofrece mitoxantrone como rescate ya que su eficacia en esta situación es muy limitada y beneficia a menos del $15 \%$ de los pacientes. En nuestros centros en la actualidad se está desarrollando un ensayo aleatorizado fase III en que se compara satraplatino, un derivado oral del platino, más prednisona versus prednisona sola.

El tratamiento del CPHR es multidisciplinar y supone un reto. El urólogo es el facultativo que vive con el paciente la mayor parte de la historia natural del cáncer de próstata. Cuando el tumor se convierte en hormonorresistente la colaboración con el oncólogo y el radioterapeuta es fundamental. El urólogo debe conocer en que instante es precisa la aportación de otras especialidades. Esto será fundamental para intentar lograr el tratamiento idóneo para cada paciente. 


\section{REFERENCIAS}

1. Huggins C, Hodges CV. Studies on prostate cancer: I. The effect of castration of estrogen and of androgen injection on serum phosphatase in metastatic carcinoma of the prostate. Cancer Res 1941;1:293-297.

2. Crawford ED, Boccon-Gibod L, Buchorsky N, Iversen P, Kirk D, Kittamura t, et al. Management of newly-diagnosed metastatic prostate cancer. In: Prostate Cancer. $2^{\text {nd }}$ International consultation on Prostate Cancer. Edited by: G.Murphy, S.Koury, L.Denis. United Kingdom: Health Publication, p353-394,1999.

3. Maximum androgen blockade in advanced prostate cancer: an overview of the randomised trials. Prostate Cancer Trialists' Collaborative Group. Lancet. 2000 Apr 29;355(9214):1491-14988.

4. Debruyne FMJ, Akaza H., Fitzpatrick JM, Mahler C, Motta M, Thraser JB, et al. Secondary hormonal therapy in prostate cancer. In Prostate Cancer. $2^{\text {nd }}$ International consultation on Prostate Cancer. Edited by: G.Murphy, S,Koury., L.Denis. United Kingdom: Health Publication, p395-405, 1999.

5. Allepuz C, Gil J, Gil P. Tratamiento del cáncer de próstata hormonorresistente. Tema monográfico del LXVI Congreso Nacional de Urología. Madrid: Ene Ediciones. 2001.

6. Heidenreich A, von Knoblock R, Hofmann R. Curren status of cytotoxic chemoterapy in hormonerefractory prostate cancer. Eur Urol 2001;39(2)121-130.

7. Dawson NA. Apples and oranges: Building a consensus for standarized eligibility criteria and end points in prostate cancer clinical trials. J Clin Oncol 1998;16(10):3398-405.

8. Petrylak DP, Tangen CM, Hussain MH, Lara PN Jr, Jones JA, Taplin ME, Burch PA, Berry D, Moinpour C, Kohli M, Benson MC, Small EJ, Raghavan D, Crawford ED.Docetaxel and estramustine compared with mitoxantrone and prednisone for advanced refractory prostate cancer. N Engl J Med 2004;351 (15):1513-1520.

9. Tannock IF, de Wit R, Berry WR, Horti J, Pluzanska A, Chin $\mathrm{KN}$ et al. Docetaxel plus prednisone or mitoxantrone plus prednisone for advanced prostate cancer. N Engl J Med 2004;351(15):1502-1512.

10. Scher HI, Steineck G, Kelly WK. Hormone-refractory (D3) prostate cancer: refining the concept. Urology 199546 (2);142-148.

11. Bubley GJ, Carducci M, Dahut W, Dawson N, Daliani D, Eisenberger $\mathrm{M}$ et al. Eligibility and response guidelines for phase II clinical trials in androgen-independent prostate cancer: recomendations from Prostate-Specific Antigen Working Group. J Clin Oncol 1999(17):3461-3467.

12. Deniel JG, Kantoff PW. Indicadores pronósticos en el cáncer de próstata refractario a hormonas. Urologic Clinics of North America 1999 Vol 2: 329-336,. Edición española.

13. Fossa SD, Paus E, Lindegaard M, Newling DW.Prostate specific antigen and other prognostic factors in patients with hormoneresistant prostatic cancer undergoing experimental treatment. Br J Urol 1992);69(2):175-179.

14. Akimoto S, Masai M, Akakura K, Shimazaki J. Tumor marker doubling time in patients with prostate cancer: determination of prostate-specific antigen and prostatic acid phosphatase doubling time. Eur Urol 1995;27(3):207-212.

15. Carducci MA, De Wesse TL, Nelson JB. El antígeno específico de la próstata y otros marcadores de la respuesta terapéutica. Urologic Clinics Of North America, 1999 Vol (2).317-328. Edicion española.

16. Kelly WK, Scher HI , Mazumdar M, Vlamis V, Schwartz, Fossa JD.. Prostate-specific antigen as a measure of disease outcome in metastatic hormone-refractory prostate cancer. J Clin Oncol 1993;11(4):607-615

17. Oh WK, Kantoff PW.Management of hormone refractory prostate cancer: current standards and future prospects. J Urol. 1998;160(4):1220-1229.
18. Klugo RC, Farah RN, Cenry JC.. Bilateral orchiectomy for carcinoma of the prostate: response of serum testosterone and clinical response to strogen therapy. Urology 1981;17(1):49-50.

19. Resse DM, Small EJ. Manipulaciones hormonales secundarias en el cáncer de próstata refractario a hormonas. Urologic Clinics of North America.1999 (2):337-347. Edicion española.

20. Smith DC, Dawson NA, Trump DL. Management of hormonerefractory disease. Secondary hormonay manipulation.. in: Comprehesive textbook of genitourinery oncology. Edited by: NJ Vogelzang, PT Scardino, WV. Shipley, DS Coffey. Philadelphia: Lippincott Williams and Wilkins, p855-869,2000.

21. Olson KB, Pienta J. Tratamiento del cáncer de próstata refractario a la hormonoterapia. AUA Update Series. Lección 4: 2000:23-29,.Versión en español.

22. Scher HI., Kelly WK. Flutamide withdrawal syndrome: its impact on clinical trials in hormone-refractory prostate cancer. J Clin oncol 1993;11(8):1566-1572.

23. Small EJ, Vogelzang NJ. Second-line hormonal therapy for advanced prostate cancer: a shifting paradigm. J Clin Oncol 1997;15(1):382-388.

24. Small EJ, Halabi S, Dawson NA, et al. Antiandrogen withdrawal alone or in combination with ketoconazole in androgenindependent prostate cancer patients: a phase III trial (CALBG 9583). J Clin Oncol 2004;22(6):1025-1033.

25. Nieh PT. Withdrawal phenomenon with the antiandrogen Casodex. J Urol 1995;153(3 pt2):1070-1072.

26. Gomella LG, Ismail M, Nathan FE. Antiandrogen withdrawal syndrome with nilutamide. J Urol 1997;157 (4):1366.

27. Dawson NA, McLeod DG. Dramatic PSA decline in response to discontinuation of megestrol acetate in advanced prostate cancer: Expansion of the antiandrogen withdrawal syndrome. J Urol 1995 (6);153:1946-1947.

28. Bissada NK, Kaczmarek AT. Complete remission of hormonorrefractory adenocarcinoma of the prostate in response to withdrawal of diethylstilbestrol. J Urol 1995;153(6):1944-1945

29. Small EJ, Srinvas S. The antiandrogen withdrawal syndrome: Experience in a large cohort of unselected patients with advanced prostate cancer. Cancer 1995;76(8):1428-1434.

30. Fowler JE, Pandey P, Seaver LE, Feliz TP. Prostate specific antigen after gonadal withdrawal and deferred flutamide treatment. J.Urol 1995;154 (2 Pt1):448-453.

31. Joyce R, Fenton MA, Rode P, Constantine M, Gaines L, Kolvenbag $\mathrm{G}$ et al. High dose bicalutamide for androgen independent prostate cancer: effect of prior hormonal therapy. J.Urol 1998159(1):149-153.

32. Scher HI, Liebertz C, Kelly WK, Mazumbar M, Brett C, Schwartz L et al. Bicalutamide for advenced prostate cancer: the natural versus treated history of disease. J Clin Oncol 1997; 15(8):2928-2938.

33. Osborn JL, Smith DC, Trump DL. Megestrol acetate in the treatment of hormone refractory prostate cancer. AmJ Clin Oncol 1997;20(3):308-310

34. Sartor O, Cooper M., Weinberger M, Headle D, Thibault A, Tompkins A, et al. Surprising activity of flutamide withdrawals when combined with aminoglutetimide, in treatment a hormonerefractory prostate cancer. J Natl Cancer Inst. 1994 2;86(3):2227. Erratum in: J Natl Cancer Inst 1994 Mar 16;86(6):463.

35. Trump DL, Havlin KH, Messing EM, Cummings KB, Lange PH, Jordan VC. High dose ketoconazole in advanced hormonerefractory prostate cancer: endocrinologic and clinical efects. J Clin Oncol 1989;7(8):1093-1098 .

36. Small EJ, Baron AD, Fippin L, Apocada D.. Ketoconazole retains activity in advanced prostate cancer patients with progresion despite flutamide withdrawal.J Urol 1997;157(4):12041207. 
37. Harris KA, Small EJ, Frohlich MW. Prospective trial of low dose ketoconazol therapy in patients with androgen-independent prostate cancer (abstract). Proc Am SoC Clin Oncol 2001;20: 2419.

38. Scholtz M, Jennrich R, Strum S, Brosman S, Johnson H, Lam R. Long-term outcome with androgen independent prostete cancer treated with ketoconazole and hidrocortisone. J Urol 2005;173(6):1947-1952.

39. Tannock I.,Gospodarovicz M, Meakin W, Panzarella T, Stewart L, Rider W. Treatment of metastatic prostate cancer with low dose prednisone: Evaluation of pain and quality of life as pragmatic indices of response. J Clin Oncol 1989(5);7:590-597.

40. Sartor O, Weinberger M, Moore A, Li A, Figg WD. Effect of prednisone on prostate-specific antigen in patients with hormonerefractory prostate cancer. Urology 1998 (2);52:252-256.

41. Smith DC, Redmen BG, Flaherty LE, Li L, Strawderman M, Pienta KJ. A phase II trial of oral diethylstilbestrol as a second-line hormonal agent in advanced prostate cancer Urology 1998;52(2):257-260.

42. Orlando M, Chacon A, Salum G, Chacon DR. Low dose continous oral fosfestrol is higly active in hormone refractory prostate cancer. Ann Oncol 2000;11(2):177-181.

43. Droz JP, Kattan J, Bonnay M, Chraibi Y, Bekradda M, Culine S. High dose continous-infusion fosfestrol in hormone-resistant prostate cancer. Cancer. 1993;71. (3 Suppl):1123-1130.

44. Ahmed M, Chksy S, Chilton P, Munson KW, Williams JH. High dose intravenous estrogen (fosfestrol) in the treatment of symptomatic, metastatic, hormone-refractory carcinoma of the prostate. Int Urol Nephrol 1998; 30(2):159-164.

45. Hedlund PO, Kenriksson P and the Scandinavian Prostate Group (SPGC) 5-Trial Study. Parenteral strogens versus total androgen ablation in the trewatment of advanced prostate carcinoma: efects on overall survival and cardiovascular mortality. Urology 2000;55 (3):328-333.

46. Yagoda A, and Petrylak D. Cytotoxic chemotherapy for advanced hormone-resistant prostate cancer. Cancer 1993;71(3 Suppl) :1098-1099.

47. Tannock IF, Osoba D, Stockler MR, Ernst DS, Neville AJ, Moore MJ, et al. Chemotherapy with mitoxantrone plus prednisone or prednisone alone for symptomatic hormonorresistant prostate cancer: a Canadian randomisad tria with paliative end points. J Clin Oncol 1996;14(6):17561764.

48. Hartley-Asp B, Natale RB, Dreicer R, Falcon S, Ricardez A, Redfern $\mathrm{C}$ et al. Phase II study of weekly intravenous estramustine phosphate $2.000 \mathrm{mg} / \mathrm{m}^{2}$ in patients with hormonerefractory prostate cancer. Proc Am Soc Clin Oncol 2001;20 183a.

49. Morote J, Lopez-Pacios MA, Ahmad A, Vila J, De Torres JA. Tratamiento del cáncer de próstata hormonorrefractario con fosfato de estramustina. Actas Urol Esp 1991;15 (5):421-424.
50. Hudes G, Einhorn L, Ross E, Balsham A, Loehrer P, Ramsey H, Sprandio J, Entmacher M, Dugan W, Ansari R, Monaco F, Hanna M, Roth B.1. Vinblastine versus vinblastine plus oral estramustine phosphate for patients with hormonorrefractory prostate cancer: a Hoosier Oncology Group and Fox Chase Network phase III trial. J Clin Oncol. 1999;17(10):3160-3166

51. Sweeny CJ, Monaco FJ, Jung SH, Wasielewsky MY, Picus J, Ansar RH. A phase II Hoosier Oncology Group study of vinorelbine and estramustine phosphate in the hormone-refractory prostate cancer. Ann Oncol 2002;13(3):435-440.

52. Triverdy C, Redman B, Flaherty LE, Kucuk O, Du W, Heilbrun LK et al. Weekly 1 hour infusion of paclitaxel: clinical feasibility and eficacy in patients with hormone-refractory prostate carcinoma. Cancer 2000;89:431-436.

53. Picus J, and Schultz M. Docetaxel (Taxotere) as a monotherapy in the treatment of hormone-refractory prostate cancer:preliminary results. Semin Oncol 1999;26:14-18.

54. Berry W, Dakhil S, Gregurich MA, Asmar L. Phase II trial of single-agent weekly docetaxel in hormone-refractory, simptomatic, metastatic carcinoma of the prostate. Semin Oncol 2001;28:815.

55. Beer TM, Pierce WC, Lowe BA, Henner WD. Phase II study of weekly docetaxel in simptomatic androgen-independent prostate cancer. Ann Oncol 2001;12:1273-1279.

56. Petrylak DP, Macartur R, O,Connor J, Shelton G, Weitzman A, Judge $\mathrm{T}$ et al. Phase II studies of docetaxel (taxotere) combined with estramustine in men with hormone-refractory prostate cancer. Semin Oncol 1999;26:28-33.

57. Petrylak.DP. The current role of chemotherapy in metastatic hormone-refractory prostate cancer. Urology 2005;65 (suppl 5A):3-8.

58. Clarke NW, Wylie JP. Chemotherapy in hormone refractory prostate cancer: where do we stand? Eur Urol 2004;46:709711.

59. Huguet J, Maroto P, Sancho G, Salvador J, López JJ, Villavicencio $H$. Docetaxel en cáncer de próstata hormonorrefractario: Análisis de respuesta y toxicidad e un grupo o seleccionado de pacientes. XXI Reunión del grupo de Urología Oncológica. Córdoba 19-20 Marzo 2004.

60. Huguet J, Maroto P, Sancho G, Segarra J, Palou J, Villavicencio H. Docetaxel en cáncer de próstata hormonorrefractario: Análisis de respuesta y toxicidad en un grupo no seleccionado de pacientes. Actas Urol Esp 2005;29 Supp:72.

Dr. J. Huguet Pérez

Servicio de Urología. Fundació Puigvert

Cartagena 340 - 08025. Barcelona

E.mail: jhuguet@fundacio-puigvert.es

(Trabajo recibido el 24 de octubre 2005) 